\title{
SEISMIC EVIDENCE FOR A WEAK BASAL LAYER DURING THE 1982 SURGE OF VARIEGATED GLACIER, ALASKA, U.S.A.
}

\author{
By MARK A. RICHARDs*
}

(Seismological Laboratory, California Institute of Technology, Pasadena, California 91125, U.S.A.)

ABstract. Wide-angle seismic reflections from a glacier bed are sensitive to the presence of subglacial water and to the mechanical strength of the basal zone. The phase of a compressional to shear ( $\mathrm{P}-\mathrm{SV}$ ) converted wave is particularly sensitive to the shear coupling between the glacier and its bed. Both shear (SV) and compressional (P) reflections from the bottom of Variegated Glacier were obtained before the 1982-83 surge event using explosive sources, and the phases of these reflections (relative to the source) were positive as expected for an ice/bedrock interface. During surge, P-wave reflections from the deepest part of the glacier bed were phase reversed, while somewhat shallower P-wave reflections were not. SV reflections were not clearly observed during surge and cannot be interpreted with confidence, even though the experiments were designed to detect them using threecomponent geophones. The unexpected P-wave phase reversal might be explained by a thin, mechanically weak or fluidized basal debris layer during surge which acts as a strong seismic absorber and suppresses multiple reflections within the layer. However, the required amount of seismic attentuation in the layer seems unreasonably large. An alternative explanation requiring $\sim 4-8 \mathrm{~m}$ or more of water or water-saturated debris (without strong attenuation) is implausible.

\section{INTRODUCTION}

The mechanical properties of the basal zone of a glacier or an ice sheet are important in determining the type of flow observed. The surface velocities measured on normal temperate valley glaciers are due both to internal deformation and to a variable amount of sliding motion at the base (e.g. Paterson, 1981, p. 71). Surge-type glaciers periodically exhibit pulses of basal sliding (reduced basal friction) one to two orders of magnitude greater than normal surface velocities. For Variegated Glacier, Alaska, these pulses were observed at intervals averaging about 17-20 years since 1906 (Tarr and Martin, 1914; Post, 1969). The map view of Figure 1 shows the geometry of the upper $12 \mathrm{~km}$ of this valley glacier and its tributaries.

An intensive study of Variegated Glacier was begun in 1973 (Bindschadler and others, 1977) and continued through the most recent surge event during 1982-83. On the basis of detailed surveying and bore-hole measurements (1979-83), $\mathrm{Kamb}$ and others (1985) concluded that surge-type velocities were caused by pulses in basal water pressure to near or slightly greater than the ice-overburden pressure; parts of the glacier were almost floated during surge. From 1979 to 1982 , B. Kamb and H. Engelhardt (unpublished manuscript) observed four to five "mini-surge" events per summer, with increasing intensity, during which the normal surface velocity of about $50 \mathrm{~cm} / \mathrm{d}$ at mid-glacier was observed to increase rapidly to $100-300 \mathrm{~cm} / \mathrm{d}$ and gradually drop back to normal within about $10-20 \mathrm{~h}$. During these mini-surge

* Present address: Department of Geological Sciences, University of Oregon, Eugene, Oregon 97403, U.S.A. events, slightly premonitory basal water-pressure pulses to near-overburden pressure were observed in basally connected bore holes, and a maximum of about $10 \mathrm{~cm}$ of surface uplift was measured as the mini-surge "front" passed by surveying stations on the glacier. The simultaneous flow velocity, water pressure, and uplift anomalies are thought to be symptomatic of the formation of water-filled cavities at the glacier bed (Kamb and others, 1985). These observations and their phenomenological explanation are compatible with some theories of glacial surges (Weertman, 1969; see Paterson (1981) for a brief review), although the fundamental cause of the surge instability (why one particular glacier surges and otherwise similar glaciers do not) remains illusive at the present time. So far, the evidence cited for extensive basal cavitation during the surge has been indirect and inferential, compelling as it may be.

In 1981, I began a series of seismic reflection experiments on Variegated Glacier to detect the presence of water-filled cavities and, more generally, to probe the changes in basal mechanical properties during surge events. The experiments described in this paper consist of seismic reflection shots both during the pre-surge summer of 1981 and during the onset of the main surge in the upper part of the glacier in 1982. Wide-angle (sub-refraction) receiver offsets were used in order to observe changes in both reflected compressional (P) waves and reflected shear (SV) waves from the glacier bed. The theoretical relationship between basal conditions and the character of seismic reflections is relatively simple in concept but somewhat complicated when it is applied to a realistic model of cavitation. In this paper I first develop a "practical" theory and experimental design for detection of abnormal basal conditions such as shear decoupling and/or subglacial water. I then discuss the experimental results from Variegated Glacier and their implications for basal conditions before and during surge.

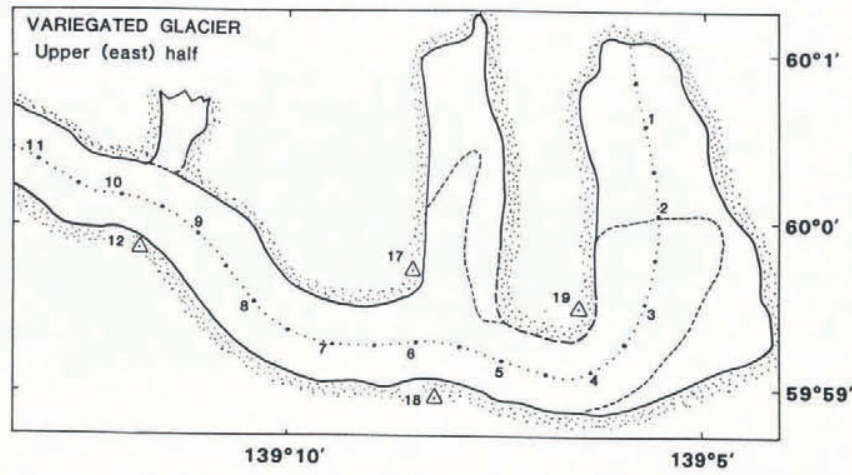

Fig. 1. Map view of the upper $12 \mathrm{~km}$ of Variegated Glacier and its main tributary. The terminus is towards the west (left side of figure), and distance in kilometers along the center line is shown by the numbered dots. Fixed survey points, indicated by triangles, are shown for reference. All areas down-glacier of the dashed lines participated in the main surge event of 1983. 


\section{BASAL REFLECTION OF ELASTIC WAVES}

Glaciologists have used seismic reflection techniques mainly for locating the bottom of ice sheets and glaciers, but more recently radio echo-sounding (Robin, 1975) has become the preferred method for mapping the glacier bed because of its relative economy. Seismic techniques have also been used to detect internal layers and to measure anisotropy in ice (Bentley, 1971, 1975). Bentley and Clough (1972) and Dewart (1976) found evidence for subglacial wet zones in West Antarctica on the basis of phase reversals and low seismic reflectivity observed in reflection profiles. The physical rationale for this interpretation can be easily understood by considering the expression for the reflection coefficient for a compressional (P) wave in medium 1 (ice) normally incident upon medium 2 (substratum):

$$
R=\frac{\rho_{2} \alpha_{2}-\rho_{1} \alpha_{1}}{\rho_{2} \alpha_{2}+\rho_{1} \alpha_{1}}
$$

where $\rho_{i}$ is the density and $\alpha_{i}$ is the P-wave velocity of medium $i$. The acoustic impedance is defined as $\rho_{i} \alpha_{i}$, so reflections are "in phase" if the impedance of medium 2 is greater than that of medium 1 and phase-reversed otherwise.

The impedance of most rocks is much higher than that of ice, so for dry basal conditions reflections should be in phase with the source. However, the impedance of water $\left(\rho_{\mathrm{w}}=1.0 \mathrm{~g} / \mathrm{cm}^{3}, \alpha_{\mathrm{y}}=1.5 \mathrm{~km} / \mathrm{s}\right)$ is much less than that of ice $\left(\rho_{\text {ice }}=0.9 \mathrm{~g} / \mathrm{cm}^{3}, \alpha_{\text {ice }}=3.6 \mathrm{~km} / \mathrm{s}\right)$, so phase reversals or diminished reflectivity might be expected if a water layer or water pockets are present at the bed.

There are several problems which limit the usefulness of this simple approach: (1) Basal debris or morainal material (e.g. Engelhardt and others, 1978) with reduced density and elastic moduli could have either larger or smaller impedance than ice. (2) Because of multiple reflections within a basal debris layer, the thickness of this layer can strongly affect the apparent reflection properties of the interface. (3) The simple acoustic viewpoint neglects the information available from the compressional to shearwave (P-SV) conversion that occurs at non-normal incidence (see Fig. 2a). In fact, this last consideration provided much of the motivation for the seismic experiments initially, because from the theoretical standpoint the reflected SV component should be much more sensitive to the presence of a thin basal water layer than the $P$ wave. Even though the most useful observations turned out to be wide-angle Pwave reflections, the thin water layer or basal layer reflection model discussed below is important both in understanding the experimental design and in interpreting the final results.

First, we consider plane-wave reflections from a "normal" ice-over-rock interface illustrated in Figure 2a. The arrows associated with each ray path indicate the direction of first motion from an explosive source. Note that the radial motion (along the shot axis) for a $\mathbf{P}$ reflection has opposite polarity to that of an SV reflection, even though both reflections are "in phase". Figure 3a (solid lines) shows the velocity potential reflection coefficients (Aki and Richards, 1980) for both P-P and P-SV reflections as a function of take-off angle $\theta$. We have used $\rho_{\text {rock }}=$ $2.7 \mathrm{~g} / \mathrm{cm}^{3}$ and $\alpha_{\text {rock }}=4.2 \mathrm{~km} / \mathrm{s}, \beta_{\text {rock }}=2.6 \mathrm{~km} / \mathrm{s}$ (representative values for the metasediments that underlie most of the glacier) and $\rho_{\text {ice }}=0.9 \mathrm{~g} / \mathrm{cm}^{3}$ and $\alpha_{\text {ice }}=3.6 \mathrm{~km} / \mathrm{s}, \beta_{\text {ice }}$ $=1.8 \mathrm{~km} / \mathrm{s}$, where $\beta$ is the shear-wave velocity. Critical angle, $\theta_{\mathrm{c}}$, is about $58^{\circ}$, and we see that there is strong P-SV conversion for $\theta \gtrsim 20^{\circ}$. For ice over water $\left(\alpha_{w}=1.5\right.$ $\mathrm{km} / \mathrm{s}, \beta_{w}=0$ ) both $\mathrm{P}-\mathrm{P}$ and $\mathrm{P}-\mathrm{SV}$ reflection coefficients are negative $\left(180^{\circ}\right.$ out of phase with the source) as shown by the dashed lines in Figure 3a; most of the wide-angle reflected energy is SV.

Reflection coefficients for the "liquid sandwich" model shown in Figure $2 \mathrm{~b}$ can be calculated using ray theory by writing the total reflected wave field (ray) as the sum of all reflected rays from the two interfaces. For example, the total SV reflection coefficient in medium 1 (ice) is given by:

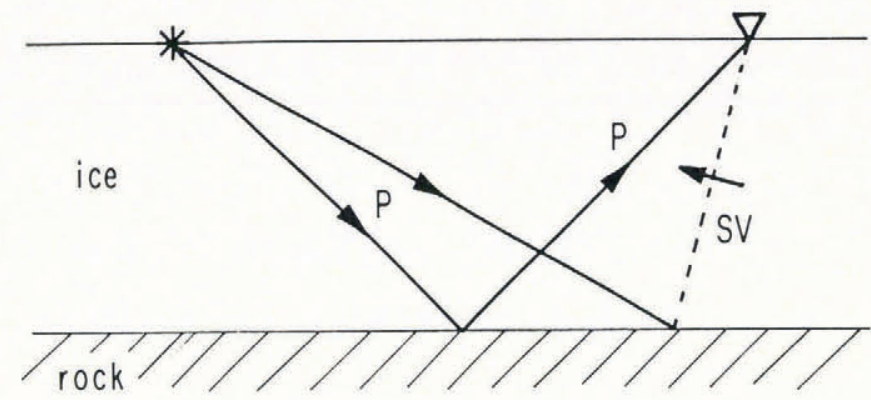

(a) Normal Conditions

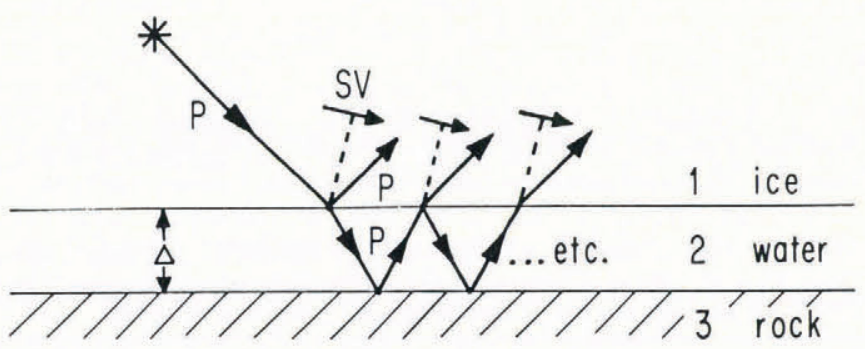

(b) Liquid Sandwich

Fig. 2. a. Diagram of compressional and shear-wave reflections from an ice/rock interface. $b$. Diagram of multiple reflections within a water layer of thickness $\Delta$ between ice and rock.

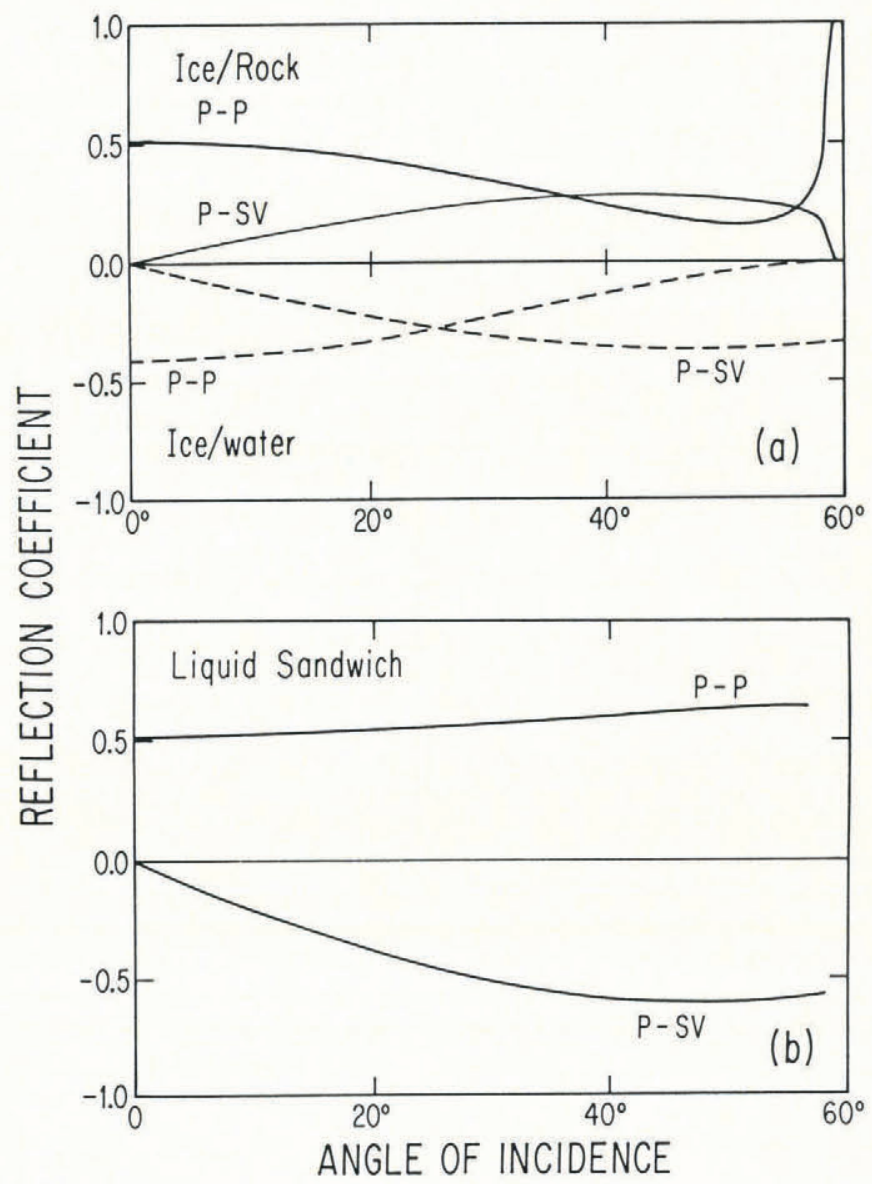

Fig. 3. a. Velocity potential plane-wave reflection coefficients for an ice/rock interface (solid lines) and for an ice/water interface (dashed lines) as functions of the angle of incidence. $b$. Reflection coefficients for $a$ very thin water layer between ice and rock. 


$$
\begin{aligned}
& A^{\mathrm{SV}}(p)=R_{12}^{\mathrm{SV}}+T_{12}^{P} R_{23}^{P} T_{21}^{\mathrm{SV}} \mathrm{e}^{-i 2 \Delta \omega \eta_{2}}+ \\
+ & T_{12}^{P}{ }_{23}^{P}{ }_{21}^{P}{ }_{23}^{P}{ }_{21}^{P} T_{21}^{\mathrm{SV}} \mathrm{e}^{-i 4 \Delta \omega \eta_{2}+\ldots \text { etc. }}
\end{aligned}
$$

where $R \mathrm{~s}$ and $T \mathrm{~S}$ are plane-wave reflection coefficients $\left(T^{\mathrm{SV}}\right.$ $=R_{23}^{\mathrm{SV}}=0$ since medium 2 is water), $\Delta$ is the thickness of the water layer (medium 2), $\omega$ is the angular frequency and

$$
\eta=\left[\left(\alpha_{2}\right)^{-2}-p^{2}\right]^{\frac{1}{2}}
$$

with ray parameter

$$
p=\frac{\sin \theta}{\alpha_{1}} .
$$

The infinite sum of Equation (2) can be written in closed form as

$$
A^{\mathrm{SV}}(p)=R_{12}^{\mathrm{SV}}+\frac{T_{12}^{P} T_{21}^{\mathrm{SV}} R_{23}^{P}}{\mathrm{e}^{i 2 \Delta \omega \eta_{2}-} R_{21}^{P} R_{23}^{P}}
$$

in which the exponential phase terms give minima and maxima depending upon the layer path length $\eta_{2} \Delta$. This interference pattern due to trapped multiples is, of course, dependent upon the frequency as well as the layer thickness. The P-P reflection coefficient can be obtained by substitution of $R_{12}^{P}$ for $R_{12}^{\mathrm{SV}}$ and $T_{21}^{P}$ for $T_{21}^{\mathrm{SV}}$ in Equation (3). Similar but more complicated expressions may be derived for a layer of basal debris with a non-zero shear modulus.

Returning briefly to consideration of the cavitation problem, it must be remembered that measured uplift (and, by inference, average cavity thickness) during mini-surge events was $\leqslant 10 \mathrm{~cm}$, which is much less than any wavelength for exploration seismic work. Therefore, the phase factors in Equation (3) are negligibly small, and there is no significant frequency dependence to be expected. For such a thin water layer, the reflection-coefficient curves of Figure $3 \mathrm{~b}$ are obtained. These curves show that, although the thin layer is essentially transparent to $P$ waves (no phase reversal), the SV waves are phase-reversed and are maximized for large source-receiver offsets. This phase reversal occurs because the intervening water layer, although having a compressional modulus of the same order as that for ice or rock, has no shear strength. (The seismic shear-wave skin depth in water is negligibly small.)

Since the water layer effectively lubricates the boundary, the curves of Figure $3 \mathrm{~b}$ could have been obtained more straightforwardly by imposing a zero shearstress boundary condition at the interface between media 1 and 3 (Miklowitz, 1978). Less idealized cases such as a soft debris layer or a water-saturated debris layer can at least be qualitatively understood (if not directly calculated) via the approach of Equation (3), and I shall return to these cases later. The two essential points to note are first that the simple approach of Equation (1) is not valid unless the immediately underlying material (water) is much thicker than the seismic wavelength. Secondly, the reflected SV wave is very sensitive to the shear coupling between ice and the substratum regardless of the layer thickness, and this is a mechanical property of direct glaciological interest.

\section{PRE-SURGE OBSERVATIONS}

Initial experiments in 1981 were designed primarily to detect wide-angle P-SV conversion from the glacier bottom. Difficulties with such an experiment are evident from the seismically determined cross-sections (Fig. 4) of the glacier in the test region (Bindschadler and others, 1977). The $\mathrm{U}$-shaped bottom, unlike the plane-layered models discussed above, causes focussing and defocussing of reflected seismic waves. Nevertheless, I found two strong, consistent reflectors in axial profile and in the area of the glacier shown in Figure 5. Good transverse seismic profiles for defining these reflectors were impossible to obtain, because the glacier was heavily crevassed towards the marginal zones. Since I was interested more in changes in wave forms rather than the detailed geometry of the reflectors, the non-ideal geometry did not turn out to be a severe restriction. (This point is discussed further below.)
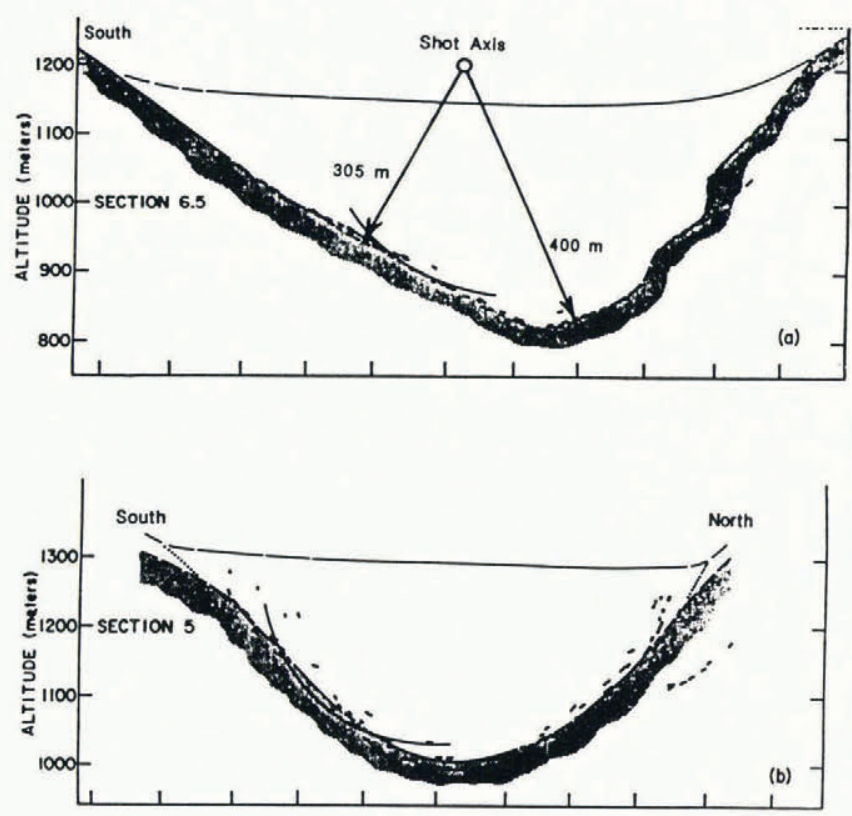

Fig. 4. Seismically determined cross-sections of Variegated Glacier at Km 6.5 and 5 (see Fig. 1) looking up-glacier. (Adapted from Bindschadler and others (1977).) In (a) the approximate shot-axis location for 1982 is shown along with arrows roughly indicating the locations of the two seismic reflectors. The shot axis is above the 1977 surface level because the glacier thickened about $50 \mathrm{~m}$ between 1977 and 1981.

A 36 station axial profile was obtained using the source-receiver geometry shown in Figure 5a. The sources were $1 \mathrm{~kg}$ Triex charges (two liquid components, detonation velocity $\sim 8000 \mathrm{~m} / \mathrm{s}$ ) shot in a bore hole about $15 \mathrm{~m}$ below the surface and well below the firn layer. Three shots of 12 stations each were recorded on paper (and photographed from video display) on a Geometrics ES1210 seismograph. Stations were spaced $20 \mathrm{~m}$ apart along the axis of the glacier, and each station consisted of a single vertical geophone (Mark Products, $10 \mathrm{~Hz}, 70 \%$ damping). The signal to noise ratio was very high, so strings of geophones were not required. Recordings of these three shots are shown in Figure 6a.

The first (P-wave) arivals (move-out velocity $\approx 3.6 \mathrm{~km} / \mathrm{s}$ ) and Rayleigh waves are obvious in the full section (Fig. 6a). Digitized versions of the 12 most distant stations (540-760 m offset) are shown in Figure $6 \mathrm{~b}$, and two faster move-out $P$-wave reflections are easily identified. These seismograms demonstrate the quality of data which can be produced on ice, and this high quality allows some interpretation of the details of the arrival wavelets. Note that the source wavelet (first arrival) and the reflection pulses are similar, with a high-frequency pulse upward (downward on seismogram) followed by a longer (lower-frequency) downward pulse and more low-frequency swings. The source and the reflections appear to have approximately the same phase, consistent with a bottom bedrock reflector and normal basal conditions. 

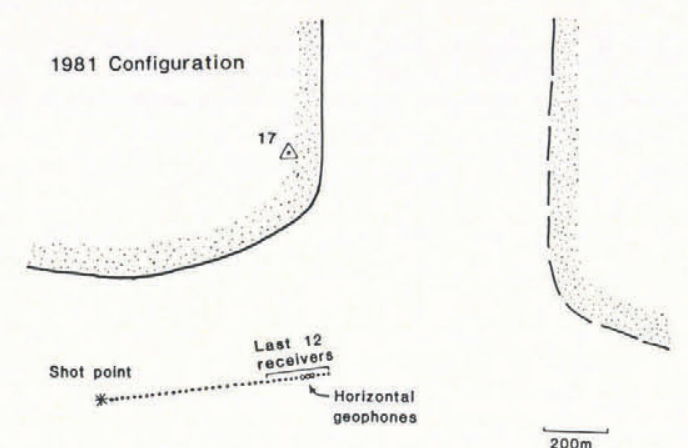

$200 \mathrm{~m}$

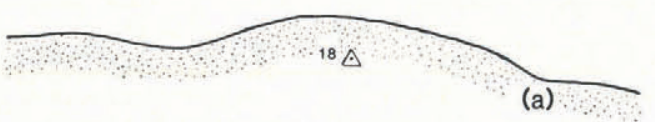

(a)

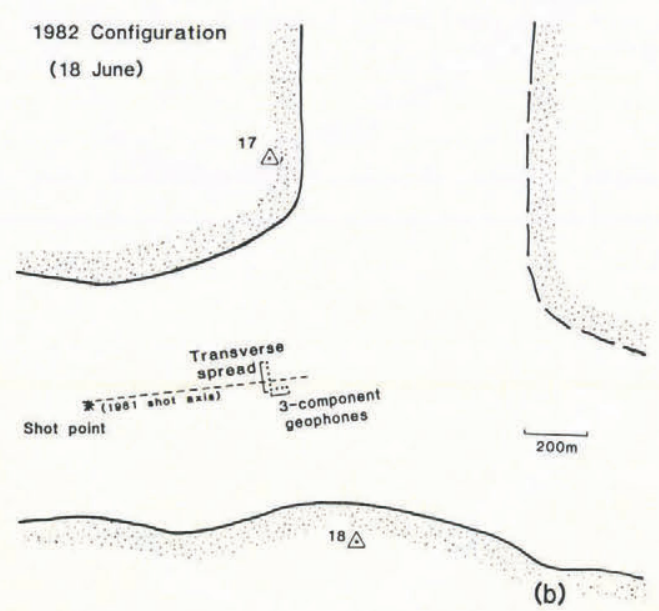

Fig. 5. a. Geometry for seismic source and receivers in the summer of 1981. Solid dots show the 36-phone (three-shot) vertical component spread (see Fig. 6). Open circles show the locations of the horizontal geophone stations. Survey points 17 and 18 are for reference to Figure 1, For the horizontal component shot, the source was $50 \mathrm{~m}$ to the south of the indicated shot point.

b. Shooting geometry for 18 June 1982. Shots of 13 and 16 June are displaced approximately $25 \mathrm{~m}$ and $10 \mathrm{~m}$ up-glacier. The source-receiver axis of 1981 is shown by a dashed line for comparison.

The travel-time plot of Figure 7 shows that the two reflected arrivals, $\mathrm{P}-\mathrm{P} 1$ and $\mathrm{P}-\mathrm{P} 2$, correspond in timing and in move-out to bottom (or side-wall) reflectors that are about $305 \mathrm{~m}$ and $400 \mathrm{~m}$, respectively, away from (perpendicular distance) the shot-receiver axis. (More will be said about the nature of these reflectors in the next section. For now it suffices to assume that they are relatively smooth features along the length of the glacier bed.) The first 24 stations also detect these two reflectors, although the display-trace amplification in the nearest offset shot record shown is too small to show the arrivals.

In order to detect $\mathrm{P}-\mathrm{SV}$ converted waves for the larger offset stations, another shot was recorded using both transversely and radially (along axis) oriented horizontal $(10 \mathrm{~Hz})$ geophones at the three stations marked by open circles in Figure 5a. (The shot point in this case was about $50 \mathrm{~m}$ due south of that shown.) Failure of the paper printer device on the seismograph, due to wet conditions, required makeshift photography of the video display screen using a $35 \mathrm{~mm}$ camera. The video display shows either the first half of the 12 channel traces or the full record with half the resolution along the time axis. (The actual digital sample rate was $1024 / \mathrm{s}$, but the video-display rate was somewhat lower.) Both the half (better time resolution) and full records for the radial components (hand-digitized and replotted) are shown in Figure 8a and b, respectively.

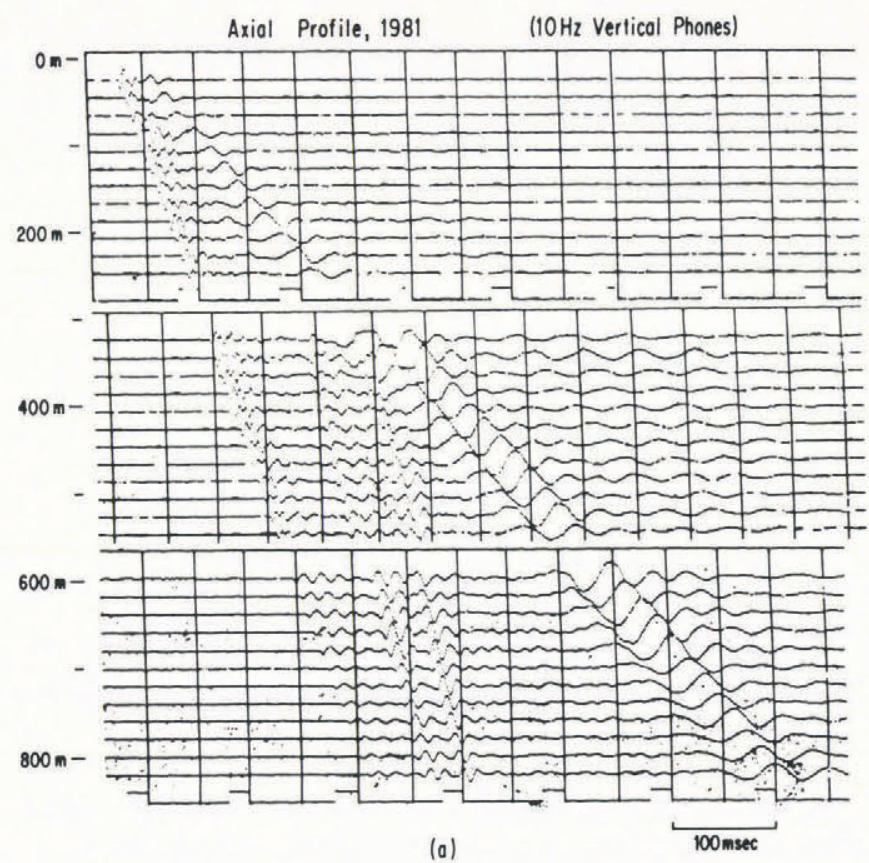

Axial Profile, $1981 \quad$ (Last 12 stations)

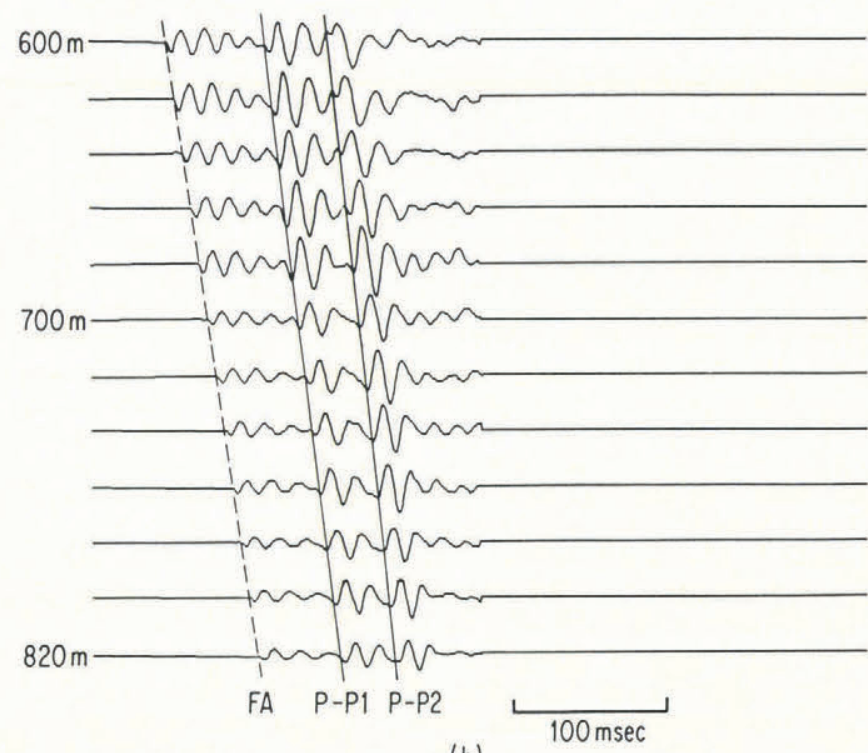

(b)

Fig. 6. a. Full 36-phone vertical component record section from three shots in 1981 (photocopy reproduction from paper-printer records). Source-receiver offset is shown on the vertical axis, time on the horizontal axis.

b. Vertical component seismograms from 12 most distant receivers shown in Figure 5. Records were hand-digitized and replotted from records similar to those of Figure 6. Onsets of first arrivals (FA) and two compressional wave reflections $(P-P 1$ and $P-P 2)$ are approximated by move-out lines.

There is a distinct phase (Fig. 8a) on the radial components which arrives after the first arrival and the two reflected $P$ arrivals. This phase is not evident on the vertical components (Fig. 6) and is less pronounced on the transverse components (not shown), suggesting a steeply incident SV wave. The move-out and arrival time are consistent with P-SV conversion from the shallower $305 \mathrm{~m}$ reflector (open circles, Fig. 7). On the full radial record (Fig. 8b) there is another sharp SV (radial phone) arrival just before the large-amplitude surface waves arrive. Although the onset of this phase is less distinct than the first SV arrival, its move-out and estimated arrival time are consistent, within the uncertainty of the time picks, with 


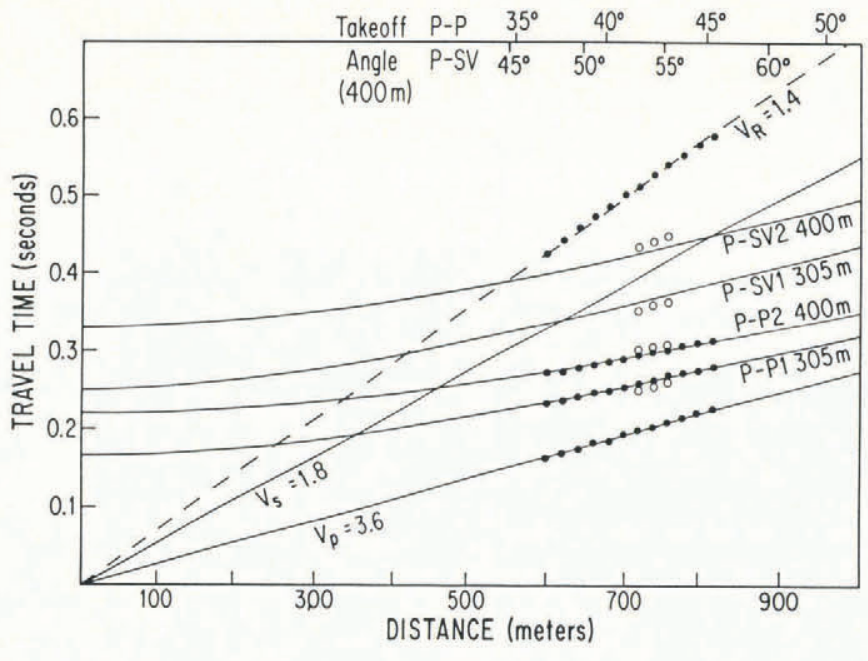

Fig. 7. Phase-arrival time picks for 36 phone spread (solid dots). Radial phone arrivals are shown by open circles. Theoretical travel-time plots for direct compressional waves (speed $3.6 \mathrm{~km} / \mathrm{s}$ ), shear waves $(1.8 \mathrm{~km} / \mathrm{s}$ ), and Rayleigh waves $(1.4 \mathrm{~km} / \mathrm{s})$ are shown along with compressional and shear-wave $(P-S V)$ reflections from $305 \mathrm{~m}$ and $400 \mathrm{~m}$ perpendicular distance from the shot axis.

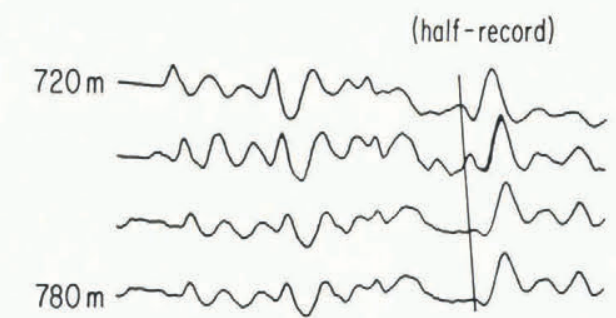

(a)

(full record)

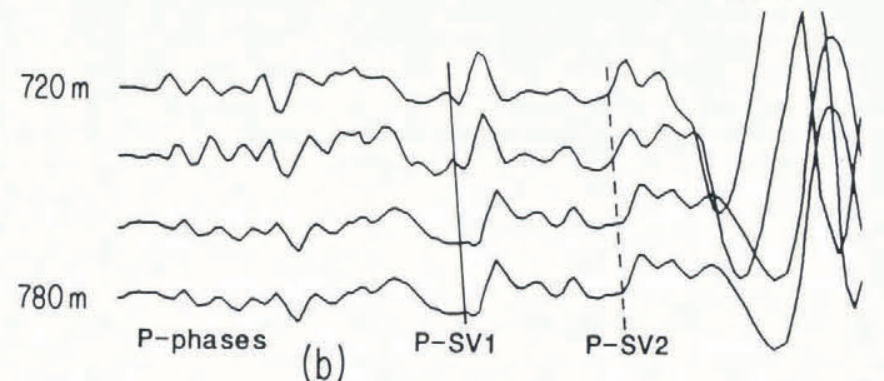

(b)

\section{$100 \mathrm{msec}$}

Fig. 8. Radial component records from horizontal component stations (Fig. 5). a. Higher resolution half records; $b$. Full-length records photographed from seismograph video display (see text).

P-SV conversion from the deeper $400 \mathrm{~m}$ reflector (open circles, Fig. 7). The $P$ arrivals from the vertical components for this shot are also shown by open circles in Figure 7. Both of the P-SV arrivals are apparently "in-phase" reflections from the ice/bedrock interface; first (highfrequency) radial motion is towards the shot point, and the large, lower-frequency pulse is of the opposite polarity as that of P-P1 and P-P2 (radial) arrivals, as expected.

Experimental conditions and time constraints did not allow further experiments during the 1981 field season, but data from these shots demonstrated the feasibility of detecting not only $\mathrm{P}$-wave reflections but also relatively clean P-SV conversions at least from the normal (nonsurging) glacier bed. The theoretical sensitivity of the P-SV polarity and amplitude to changes in basal conditions during surge encouraged a repeat of wide-angle reflection experiments during the 1982 field season in which the upper part of Variegated Glacier began to surge.

\section{OBSERVATIONS DURING SURGE}

Between 30 May and 25 June 1982 the flow velocity of the upper part of Variegated Glacier increased steadily from about $2.5 \mathrm{~m} / \mathrm{d}$ to almost $10 \mathrm{~m} / \mathrm{d}$. The glacier below $\mathrm{Km} 10$ (Fig. 1) did not experience similar increases, and on 26 June the upper-glacier velocity dropped dramatically to less than $4 \mathrm{~m} / \mathrm{d}$. Although mini-surge type events occurred sporadically in the ensuing weeks, the impressive activity prior to 26 June did not recur until the second phase (main surge event), which began in winter 1982-83 and culminated in the summer of 1983. However, Kamb and others (1985) considered the 1982 upper glacier activity to have been the true onset of the glacier surge.

Measurements of the basal water pressure in bore holes near the 1982 shot point (shown in Fig. 5b) revealed anomalously high pressure prior to 26 June. High sliding rates at the bed of the glacier due to high water pressure (relieved overburden) are implied. Ice-drilling capabilities were fortunately available at the shot-point bore-hole site used in 1981. A series of seismic reflection experiments was performed between 13 and 18 June 1982 to detect possible differences in the basal P-SV converted reflections which had been observed before surge (1981). Unfortunately, logistical and safety problems resulting from the surge made post-surge experiments at this site impossible both after 18 June 1982 and during all of the 1983 main surge event. Also, crevassing about $800 \mathrm{~m}$ up-glacier from the shot point prevented a repeat of the full $780 \mathrm{~m}$ three-component spread used during 1981. Suitable isolation from the seismic interference from this crevasse field was achieved by not deploying receivers within about $100 \mathrm{~m}$ of the crevasses.

Most of the experiments were conducted with four integral three-component $10 \mathrm{~Hz}$ geophones (comprising 12 channels of data). The seismograms were recorded digitally via a newly acquired digital tape unit, which improved the data quality. Since the maximum offset available was $700 \mathrm{~m}$, the surface waves arrived just after the expected arrival time for $\mathrm{P}-\mathrm{SV}$ from the $400 \mathrm{~m}$ reflector. Because the reflected arrivals contained mostly energy above $40 \mathrm{~Hz}$ and since the surface waves were mostly $20 \mathrm{~Hz}$ or less in frequency content, a $30 \mathrm{~Hz}$ high-pass filter was applied to each channel prior to A/D conversion (an option on the Geometrics seismograph) in order to prevent the signal from being clipped due to the large-amplitude, low-frequency surface waves. This had little effect on the bottomreflection wave forms but effectively eliminated the surface waves on the seismograms (Figs 9 and 10).

A three-component seismogram from 18 June 1982 (shot in the configuration shown in Figure $5 \mathrm{~b}$ ) is shown in Figure 9. (Note that the vertical component recording polarity is opposite to that in 1981.) The extremely low noise level is evident, along with the clean first arrival and $\mathrm{P}$-wave reflection wave forms. The four radial components from three different shots during the June 1982 surge event are shown in Figure 10a, b, and c. The first-arrival wave forms indicate the reproducibility of the experiments from shot to shot. An arrival phase, relatively prominent on the radial components, is also found with the appropriate timing and move-out for the anticipated P-SV conversion. However, this phase is not nearly as distinct as that found in 1981. Its first motion and phase relative to the first arrival is not certain, but the arrival appears to have opposite polarity from the first arrival and first $P$ reflection, i.e. the expected phase relationship for an ice/rock reflection. A consistent phase corresponding to the second P-SV arrival observed in 1981 is not evident in the radial component seismograms.

In an attempt to clarify these reflected phases, a frequency-domain deconvolution was performed using the first-arrival wavelet as an approximation to the source. Both source and seismogram were Fourier transformed and a deconvolution spectrum formed by complex division of the 


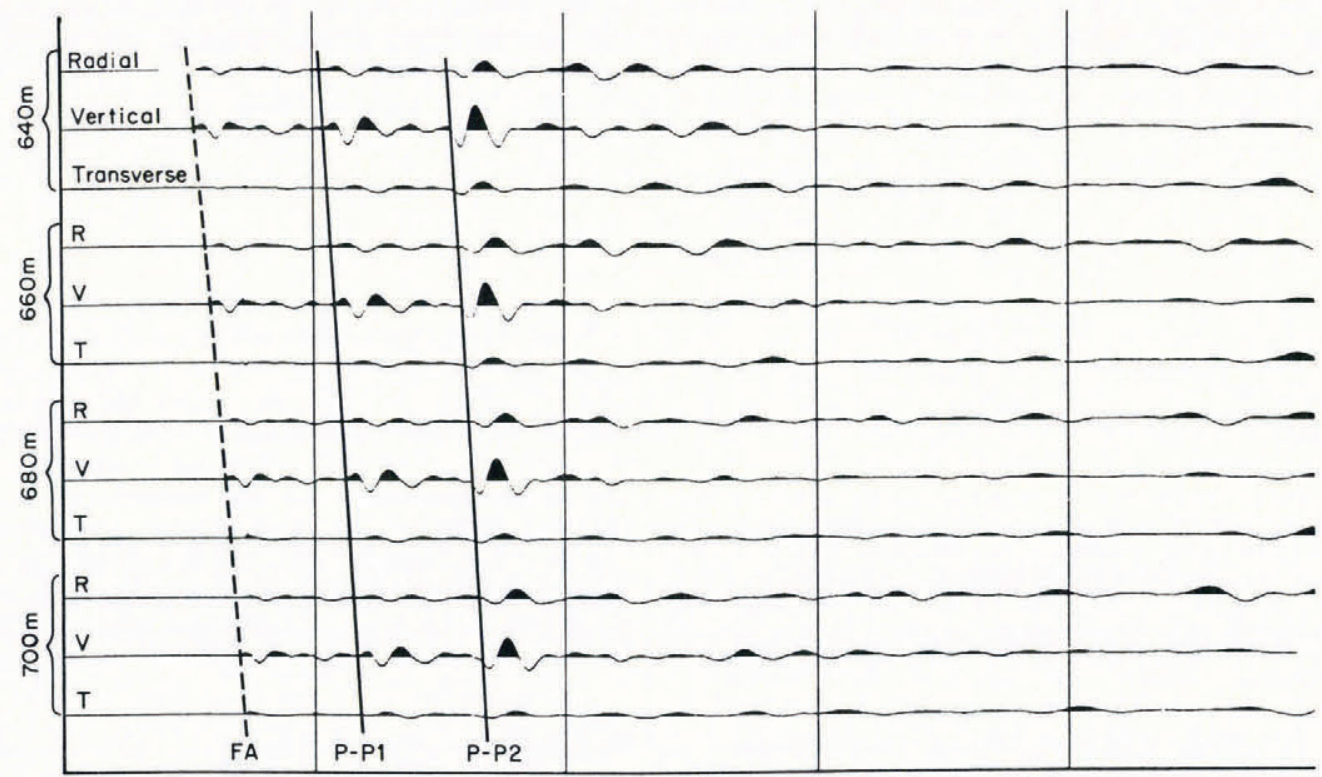

$100 \mathrm{msec}$

Fig. 9. Three-component records from 18 June from digital tape recording. "Up" on the seismograms is up, away from shot point, and towards south, respectively, for vertical, radial, and transverse components. Station locations are shown in Figure 5.

Axial Profile, 1982

13 June

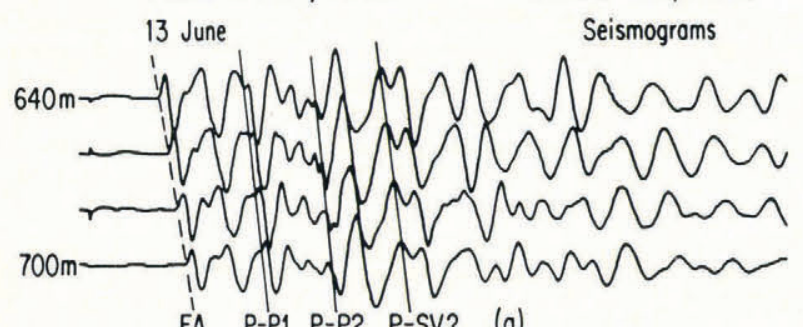

FA P-P1 P-P2 P-SV? (a)

16 June
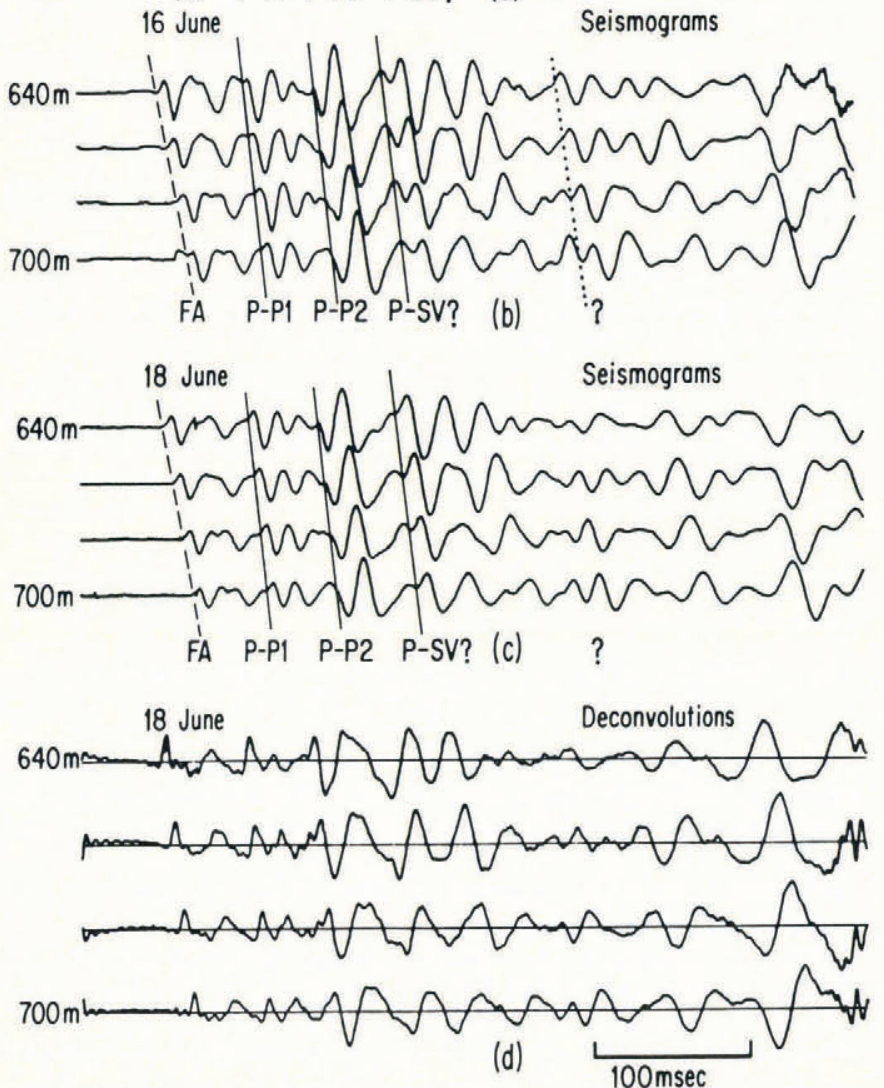

seismogram spectrum by the source spectrum. Representing the source time signal as $s(t)$, the seismogram as $h(t)$, and the "reflection filter" as $r(t)$, I used the linear convolution model

$$
h(t)=r(t) s(t)+\text { (noise) }
$$

to obtain the Fourier domain expression

$$
\hat{R}(\omega)=\frac{H(\omega)}{S(\omega)+W(\omega)}
$$

where $\omega$ is the angular frequency, $2 \pi f$, and $W(\omega)$ is a small non-zero number only when $S(\omega)$ falls below a chosen threshold value; $W(\omega)$ is often called a "water-level" parameter and serves to prevent small amounts of random noise from strongly biasing the Fourier-domain estimate $\hat{R}(\omega)$ at frequencies where both $S(\omega)$ and $H(\omega)$ are small. Typically, $W(\omega)$ is chosen to be $1-5 \%$ of the maximum of $S(\omega)$. The estimator for reflection-arrival times, $\hat{r}(t)$, is formed by an inverse Fourier transform of $\hat{R}(\omega)$. (Note that the symbol $\hat{R}$ here is not related to the symbol $R$ of Equations $(1)-(3)$.)

Deconvolutions, $\hat{r}(t)$, of the radial components of the 18 June 1982 record are shown in Figure 10d. The width of the first-arrival deconvolution peak indicates the limits on resolution in timing of the arrival using this method. The two-sided peak-with-side lobes pattern is a result of the band-limited nature of the signal. The method clearly picks the $P$ arrivals, even on the radial components, but there are no obvious P-SV arrivals. I was unable to produce any strong $\mathrm{P}-\mathrm{SV}$ arrivals during the 1982 experiments, but more discussion of this apparent failure will be delayed in order to address a more remarkable observation.

Close inspection of the deconvolutions and the seismograms themselves shows that the $\mathrm{P}-\mathrm{P} 1$ and $\mathrm{P}-\mathrm{P} 2$ arrivals are of opposite phase. Furthermore, $\mathrm{P}-\mathrm{P} 2$ is of opposite phase to the first arrival. Examination of the vertical components (where $P$ waves are clearer) in Figure 9 and Figure 11d also shows that the P-P2 wave form is phase-reversed. This

Fig. 10. $a, b$, and c. Radial components of three-component shots from three different days during the 1982 surge event. Arrival phases are indicated. $d$. Deconvolutions of seismograms in (c) using the source wavelet of the receiver at $640 \mathrm{~m}$. 
is an unexpected result as explained above. Phase reversal of the P-wave reflections was not anticipated because of the very small assumed thickness of any basal water layer or cavities during surge.

Deconvolution of the vertical seismograms shows this phase reversal clearly (Fig. 11a, b, and c) for the three June 1982 shots analysed above. The first arrival and first P-P1 reflection are almost identical, but P-P2 is between about $90^{\circ}$ and $180^{\circ}(\pi / 2$ to $\pi)$ phase-shifted. This is most clear in the 16 June shot, and the 13 June shot shows a more complicated, perhaps multiple, P-P2 arrival. The question is: are these $1982 \mathrm{P}-\mathrm{P} 2$ reflections demonstrably different from the $1981 \mathrm{P}-\mathrm{P} 2$ reflections, given the uncertainties that result from focussing and defocussing at the irregular glacier bed?

First, we return to the 1981 vertical component seismograms. Deconvolutions were performed as above, and the results for the 12 most distant channels in the 1981 axial profile are shown in Figure 12. Even though the source wavelet used was not as well defined as in 1982

Axial Profile, 1982

(Vertical components)

13 June

Deconvolutions

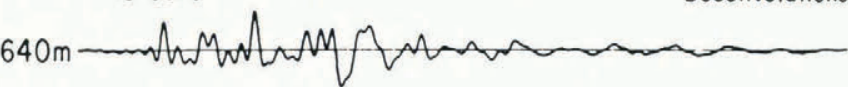

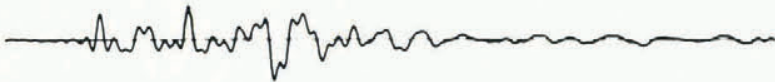

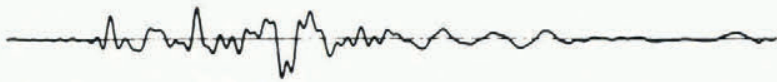

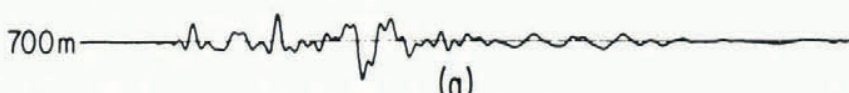

16 June

(a)

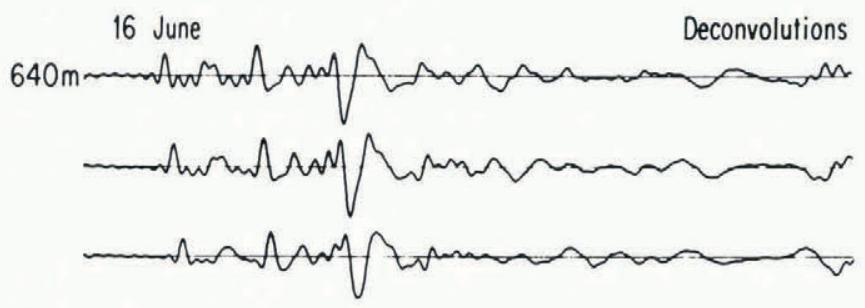

$700 \mathrm{~m} \longrightarrow$ hon

(b)

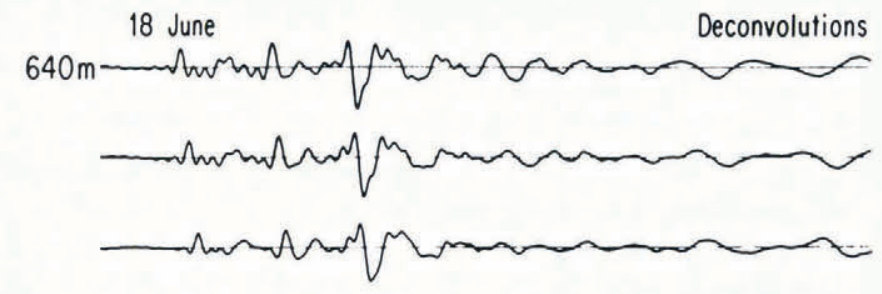

$700 \mathrm{~m}$

(c)

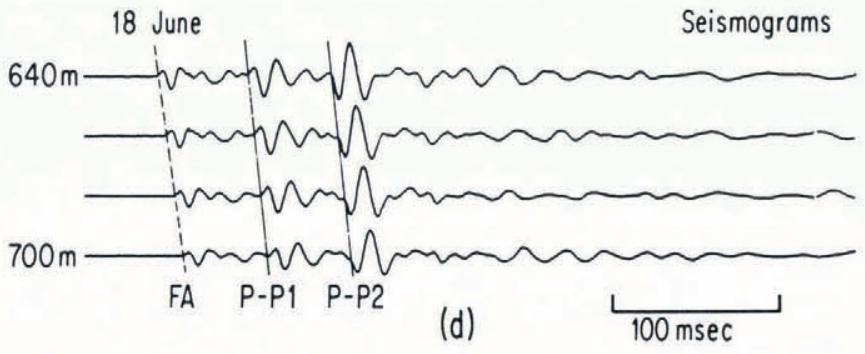

Fig. 11. $a, b$, and c. Deconvolutions of vertical components of three-component shots from three different days of June 1982. Vertical component seismograms for 18 June are shown in (d). The source wavelet for the above deconvolutions is from the receiver at $640 \mathrm{~m}$ in $(d)$.

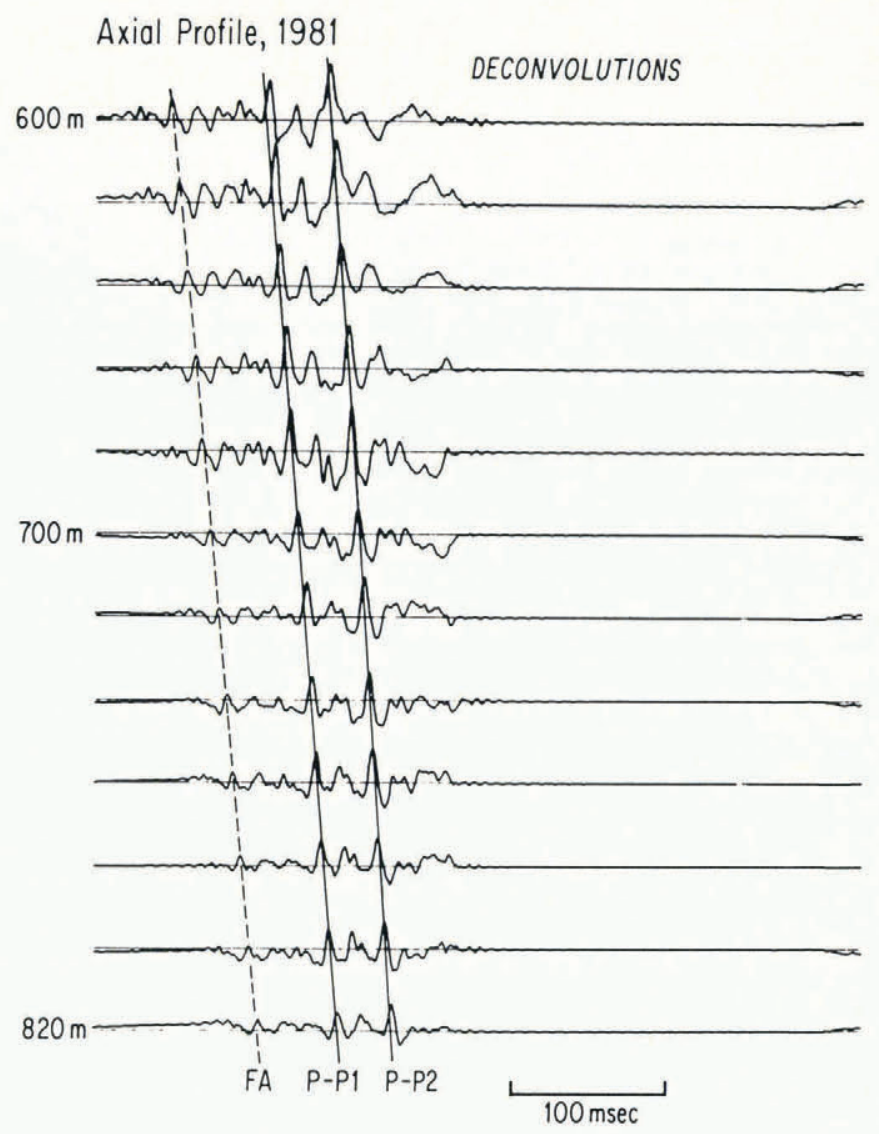

Fig. 12. Vertical component deconvolutions from 12 most distant receivers of 1981 spread (see Figs 5 and 6). Source wavelet is from channel 4 of Figure $6 b$.

(due to relatively poor recording conditions), the lack of any consistent phase shift between $\mathrm{P}-\mathrm{P} 1$ and $\mathrm{P}-\mathrm{P} 2$ is clear in Figure 12 as well as in the seismograms (Fig. 6b). The fact that this holds over a considerable distance range is impressive considering the possible irregularities of the glacial valley (basal) topography. Both of these reflectors $(305 \mathrm{~m}$ and $400 \mathrm{~m})$ must be fairly consistent features in this region of the glacier.

Much more was learned about the nature of these reflectors and about the reliability of the 1982 P-P2 phase reversal by using the transverse spread of vertical receivers shown in Figure 5b. Seismograms and deconvolutions for this spread are shown in Figure 13. The first reflection (P-P1, $305 \mathrm{~m})$ moves out rapidly from south to north, and is probably a reflection from the gently sloping south side wall (Fig. 4a). The P-P1 reflection decreases in amplitude and becomes less distinct in relative phase towards the north, also consistent with a south side-wall reflection. The $\mathrm{P}-\mathrm{P} 2(400 \mathrm{~m})$ reflection is less sensitive in arrival time to the transverse-receiver position. It is a more near-bottom reflector, probably from the deepest part of the glacier, also consistent with the Bindschadler and others (1977) profile. (In Figure $4 \mathrm{a}$, the approximate shot-axis location and radii to the inferred reflectors are shown. The shot axis is about $50 \mathrm{~m}$ above the level of the glacier, because the glacier thickened between 1977, when the profile was made, and 1982.) Deconvolution (Fig. 13b) shows that the P-P2 reflection is consistently of opposite phase to the first arrival across $100 \mathrm{~m}$ of the glacier. Intrusion towards the center of the glacier of the marginal crevasse fields to the north and south during this surge event prevented extension of this transverse profile. However, this shot shows that the 1982 $\mathrm{P}-\mathrm{P} 2$ phase reversal is not a local focussing phenomenon due to slightly differing 1981 and 1982 source-receiver configurations. It also shows that the phase reversal originates from a reflector more representative of the bottom of the glacier, as opposed to the "side-wall" character of P-P1.

This last point is further verified by the overlay of radial and transverse components (Fig. 14) from the threecomponent shot of 18 June 1982. On these seismograms "up" 
Transverse Profile, 18 June 1982 (Vertical Components)

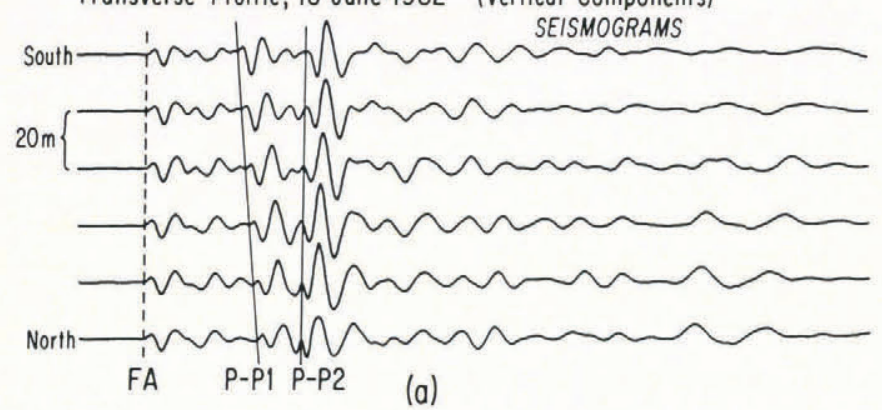

DECONVOLUTIONS
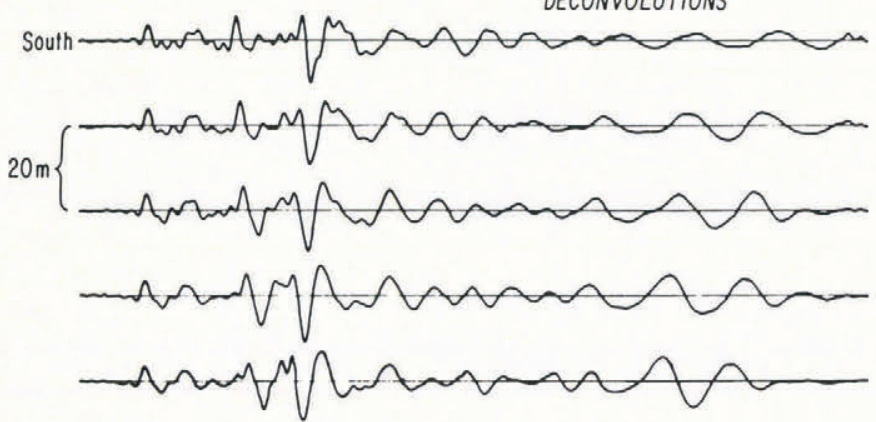

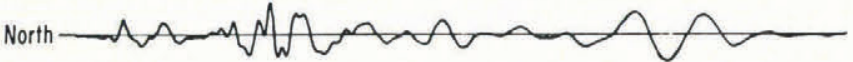

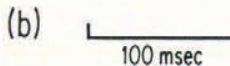

Fig. 13. a. Vertical component seismograms from transverse spread of 18 June 1982 (see Fig. 5).

$b$. Deconvolutions of transverse spread using the source wavelet from the third channel from the top of $(a)$.

Axial Profile, 18 June, 1982

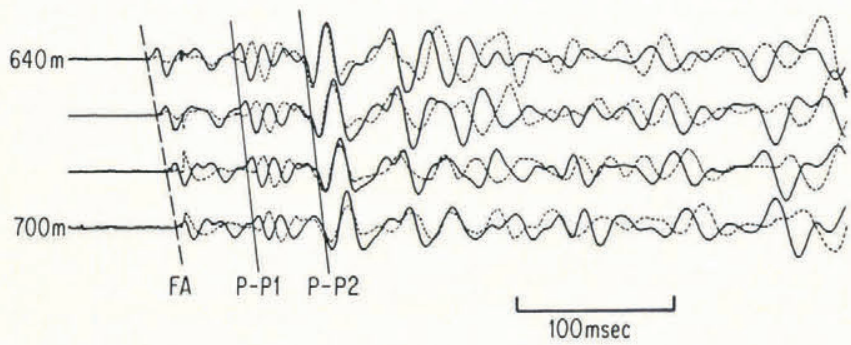

Fig. 14. Superposition of radial component seismograms and transverse components from shot of 18 June 1982. Transverse components are amplified to match the radial component amplitude for the $P-P 2$ arrival.

is radially away from the shot point (solid lines) and transversely towards the south (dashed lines). Note that there is relatively little transverse motion for the first arrival (as expected). The horizontal "particle motions" for P-P1 and $\mathrm{P}-\mathrm{P} 2$ verify the southerly and northerly origins, respectively, of these two phases. The rectilinearity of the particle motion for the two reflections is evident in Figure 14, and this relationship between the radial and transverse components provides another check on picking the onset (first motion) of the phase.

In order to check for any resolvable frequency dependence of the phase relationships discussed, I attempted to deconvolve individual $\mathrm{P}-\mathrm{P} 1$ and $\mathrm{P}-\mathrm{P} 2$ wavelets relative to each other and relative to source wavelets. This proved to be an unreliable technique, because (1) the choice of the length and onset of wavelets is somewhat arbitrary and almost guaranteed to be a biased selection, and because (2) non-zero end-point effects contaminate the higher frequencies (tapering the ends of the wavelets is also unsatisfactory). No discernible frequency dependence for the phase shift was found using individual wavelet deconvolutions. The "blind" full wave form deconvolutions of Figures 10,11, 12, and 13 are probably the least biased indicators of the sharpness (or lack thereof) of arrivals. In this respect, the P-P2 deconvolution arrival peaks in 1982 appear to be somewhat broader than the P-P1 arrivals, indicating, perhaps, less high-frequency content in the "phase-reversed" P-P2.

As noted above, the 1982 P-P2 arrivals show a shift between $90^{\circ}$ (Hilbert-transformed) and $180^{\circ}$ (phasereversed). I judge the average phase shift to be about 135$180^{\circ}$ at the dominant reflection frequencies which are generally greater than $40 \mathrm{~Hz}$. Frequencies greater than about $80-100 \mathrm{~Hz}$ have very low amplitude on all seismograms. Since the response of the geophone/seismograph system is essentially flat out to these frequencies, the experiments are limited in frequency range by the inability of either the source to generate or (more likely) the glacier ice to transmit higher frequencies. This was verified by test shots using $100 \mathrm{~Hz}$ free-period vertical geophones, which did not significantly increase the higher-frequency content of the signals recorded.

A quantitative model, including attenuation in an "average" basal layer, can be empirically constructed by modifying Equation (3) for P-wave reflection from the debris layer:

$$
A^{P}(p)=R_{12}^{P}+\frac{\left(T_{12}^{P} T_{21}^{P} \gamma R_{23}^{P}\right.}{\mathrm{e}^{i 2 \Delta \omega \eta_{2}-\gamma R_{21} R_{23}}}
$$

where $\gamma$ is the fraction of energy transmitted in two passes through the thin fluid medium. Phase shifts of $90-180^{\circ}$ can be obtained, even as $2 \Delta \omega \eta_{2} \rightarrow 0$, for values of $\gamma$ ranging from about 0.5 down to 0.1 , depending upon the density and bulk modulus of the debris layer. A value of $\gamma=0.1$ implies a very small $Q$ (e.g. Aki and Richards, 1980, p. 168) of about 0.2 for a $40 \mathrm{~Hz}$ seismic wave and a layer thickness of $10 \mathrm{~cm}$. Seismic reflection can also result from changes in $Q$ alone, even if there is no change in elastic moduli or density. For a pure $Q$ reflection the reflected signal is the Hilbert transform of the incident wave, i.e. a $90^{\circ}$ phase shift (K jartansson, 1984).

\section{SUMMARY OF OBSERVATIONS}

The principal results of the seismic experiments are summarized as follows:

(1) Two distinct reflectors are present in the area surveyed. The first is from the gently sloping south side wall and is located about $305 \mathrm{~m}$ from the shot-receiver axis. The second is about $400 \mathrm{~m}$ from the axis, slightly to the north, and is more nearly a bottom reflector.

(2) Both P-P and P-SV reflections were identified in 1981 before the onset of surge. The phases of these arrivals, relative to the direct first arrival, are consistent with reflections from an ice-rock interface (low-high impedance).

(3) During surge in 1982, the first P-SV1 phase was only weakly detected, and the second P-SV2 phase was not detected. Interference from surface waves and filtering attempts to reduce the interference may have obscured the P-SV2 phase.

(4) Also, during surge the second P-wave reflection, P-P2, was phase-shifted $\sim 135-180^{\circ}$ with respect to both the first arrival and the P-P1 reflection. Reflection signals contain mostly energy between about $40 \mathrm{~Hz}$ and $80 \mathrm{~Hz}$. Also, the P-P2 : P-P1 amplitude ratio is somewhat larger in 1982 than in 1981 .

(5) It is unlikely that the phase shift is due to irregularities in the glacier bed (geometrical distortion of the reflected wave fronts) coupled with slightly different shooting configurations. The phase relationships observed in both 1981 and 1982 persist over a fairly large aperture. 


\section{DISCUSSION}

The phase reversal of the near-bottom $\mathrm{P}$-wave reflection is an unexpected result. The observations indicate that part of the basal region underwent a dramatic change in seismic reflection properties from pre-surge to surge experiments. That the "side-wall" reflection did not change is understandable; even if high water pressure during surge caused a "thin" $(\leqslant 1 \mathrm{~m})$ layer of water between the ice and rock wall, the $\mathrm{P}$-wave reflection should be essentially unaffected. On the other hand, the deepest part of the glacier bed may behave differently during surge, having, perhaps, a thicker layer of water or water-saturated till. However, phase reversal at the glacier bed requires a basal water layer $\sim 4-10 \mathrm{~m}$ thick (one-quarter wavelength at $40-80 \mathrm{~Hz}$ ) according to the simple "liquid sandwich" model considered above. Since the uplift detected from surveying during surge is only of the order of $10 \mathrm{~cm}$, some other physical model is necessary.

An analogue to this apparent paradox is found in the "bright spots" which sometimes appear in seismic prospecting for hydrocarbons. Very large-amplitude reflections, of ten phase-reversed, indicate layers of gas, water, or petroleum saturation (Sheriff, 1980). These layers are sometimes thinner than the seismic signal wavelengths. The large amplitudes and phase reversals may represent essentially first surface reflections with little transmission of seismic energy through the strongly attenuating (very low $Q$ ) bright-spot stratum. Under such conditions, the multiples within the layer (see Equation (2)) are absorbed, and the apparent thickness of the layer is much greater. The attentuation could be due to either intergranular friction in a liquid- or gas-filled porous medium (Johnston and others, 1979), viscous dissipation due to the bulk modulus (compressibility) contrast between porous rocks and interstitial gas or liquid, or both. (Another analogue is that of common acoustic tiling, which absorbs sound wavelengths much greater than the tile thickness.)

A similar mechanism may apply to compressional wave reflections from the glacier bed during surge. Deep borehole observations (Engelhardt and others, 1978) of glacier beds consistently show a silt-gravel-cobble layer of debris between the glacial ice and the underlying bedrock, contrary to the classical polished rock interface concept. On Variegated Glacier, bore-hole measurements show that the confining pressure compacting this debris must be relieved by water (pore) pressures almost equivalent to the overburden during surge; the debris layer may be essentially fluidized much like an oversaturated sand. Consequently, a dramatic decrease in the shear strength of this layer and an increase in seismic attenuation could result during surge. If the bright-spot analogy is appropriate, then the P-P2 phase reversal is a direct indication of weakening of the basal layer during surge. This alternative model is illustrated in Figure 15.

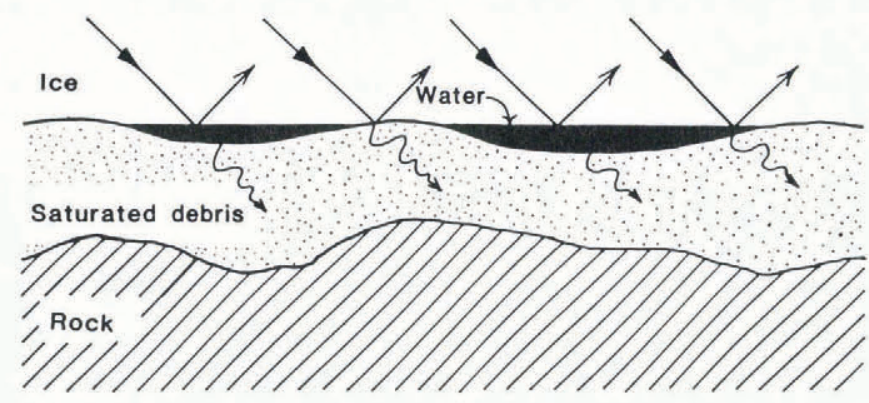

Fig. 15. Highly schematic illustration of the "first-surface" reflection of incident seismic waves and the hypothesized absorption of seismic energy in basal debris layer.

The main problem with the absorption hypothesis is that the "bright-spot" (natural gas pocket) analogy appeals to a very large contrast between the bulk moduli of a highly compressible gas and a relatively incompresible host medium, resulting in large viscous dissipation. Since the glacier bed is water-saturated during surge, this absorption mechanism is not entirely appropriate. Indeed, such large seismic absorption in a debris layer seems unlikely. Recently, Blankenship and others (1986) obtained strong reflections from both the top and bottom of a thick $(\sim 5 \mathrm{~m})$ debris layer beneath a surging ice stream in West Antarctica. If their interpretation is correct, it certainly does not imply extremely high compressional wave attenuation in the layer. Unfortunately, I have found no other alternative explanation for the phase reversal of P-P2.

The main weakness of this study is the lack of extensive coverage of the glacier bed - the P-P2 phase reversal may be a "local" phenomenon (on the scale of $\sim 100 \mathrm{~m}$ in lateral extent). However, it is unlikely that it results from geometrical effects, so the observation must be left as something still to be satisfactorily explained. Some drastic change in the mechanical properties of the deepest part of the glacier bed seems to be required.

Of course, basal cavitation (Kamb, 1970), which is thought to be an important process during surge, probably produces subglacial water patches. My inability to detect a P-SV2 converted reflection may be the result of an averaging of phase-reversed (from water patches) and unreversed (from rock patches) SV reflections at the bottom of the glacier. This would be an obvious interpretation in lieu of the P-wave reversal. However, the P-P2 reflection, by contrast, has a larger amplitude during surge, and its averaging process would have to be different. Because elastic wave reflections depend in a very complicated way upon angle of incidence and layer densities and moduli and, since the range of these properties in the basal layer is not well constrained, a more detailed theoretical analysis is not merited by the present data. An alternative explanation for the relative weakness of $\mathrm{P}-\mathrm{SV}$ phases is high shear-wave attenuation resulting from more water in the glacier itself during surge (Jacobel and Raymond, 1984).

\section{CONCLUDING REMARKS}

In wide-angle reflection experiments on the surge-type Variegated Glacier, a phase-reversed near-bottom P-wave reflection was detected during the 1982 surge event. This surprising result might be interpreted in terms of a very weak, highly attenuating, fluidized sediment or debris layer, presumably in addition to basal cavitation, resulting from high water pressure during surge. Seismic phase reversal due to water alone at the glacier bed is unlikely.

Attempts to isolate P-SV reflections were largely unsuccessful during surge, even though (1) pre-surge P-SV arrivals were clearly identified, and (2) the experiments were specifically designed to detect changes in P-SV. Conditions for these experiments were not ideal. The irregular (U-shaped) glacier bed complicates the interpretation of reflected arrivals, and logistical considerations allowed only a few useful shots. A post-surge event shot at the same location was, regrettably, not possible. However, a repeat shot is now feasible, since the glacier will not surge for almost another 20 years.

Under more optimal conditions, wide-angle seismic reflection should, theoretically, be able to detect wet or pressurized subglacial zones using P-SV arrivals on horizontal or three-component receivers. Evidently, P-wave reflections are also sensitive to surge-type basal conditions. Surging ice channels in large polar and sub-polar ice sheets overlying relatively flat-bottomed terrain would provide more suitable sites for this type of experiment. Polar-ice experiments may give much higher-frequency information and provide a better basis for modelling and understanding seismic reflection anomalies from a surging glacier.

\section{ACKNOWLEDGEMENTS}

This paper is dedicated to the late Professor C. Hewitt Dix, whose insight and patient guidance were invaluable. The field work was encouraged and generously supported by B. Kamb through a U.S. National Science Foundation grant. Field assistance from G. Humphreys, R. Svendsen, H. Aschmann, K. Echelmeyer, and B. Quinn is gratefully acknowledged. D. Helmberger and R. Clayton contributed ideas to the experimental design and data analysis. 


\section{REFERENCES}

Aki, K., and Richards, P.G. 1980. Quantitative seismology - theory and methods. San Francisco, W.H. Freeman.

Bentley, C.R. 1971. Seismic evidence for moraine within the basal Antarctic ice sheet. In Crary, A.P., ed. Antarctic snow and ice studies II. Washington, DC, American Geophysical Union, 89-129. (Antarctic Research Series 16.)

Bentley, C.R. 1975. Advances in geophysical exploration of ice sheets and glaciers. Journal of Glaciology, 15(73), 113-35.

Bentley, C.R., and Clough, J.W. 1972. Antarctic subglacial structure from seismic refraction measurements. In Adie, R.J., ed. Antarctic geology and geophysics. Symposium on Antarctic Geology and Solid Earth Geophysics, Oslo, 6-15 August 1970. Oslo, Universitetsforlaget, 683-91.

Bindschadler, R., Harrison, W.D., Raymond, C.F., and Crosson, R. 1977. Geometry and dynamics of a surge-type glacier. Journal of Glaciology, 18(79), 181-94.

Blankenship, D.D., Bentley, C.R., Rooney S.T., and Alley, R.B. 1986. Seismic measurements reveal a saturated porous layer beneath an active Antarctic ice stream. Nature, 322(6074), 54-57.

Dewart, G. 1976. Seismic evidence of a wet zone under the West Antarctic ice sheet. Journal of Glaciology, 16(74), 73-88.

Engelhardt, H.F., Harrison, W.D., and Kamb, B. 1978. Basal sliding and conditions at the glacier bed as revealed by bore-hole photography. Journal of Glaciology, 20(84), 469-508.

Jacobel, R., and Raymond, C. 1984. Radio echo-sounding studies of englacial water movement in Variegated Glacier, Alaska, U.S.A. Journal of Glaciology, 30(104), 22-29.

Johnston, D.H., Toksöz, M.N., and Timur, A. 1979. Attenuation of seismic waves in dry and saturated rocks: II. Mechanisms. Geophysics, 44, 691-711.

Kamb, B. 1970. Sliding motion of glaciers: theory and observation. Reviews of Geophysics and Space Physics, 8(4), 673-728.

Kamb, W.B., and 7 others. 1985. Glacier surge mechanism: 1982-1983 surge of Variegated Glacier, Alaska. Science, 227(4686), 469-79.

Kjartansson, E. 1984. Reflections due to contrasts in $Q$. Stanford, CA, Stanford University Press. (Stanford Exploration Project, 16.)

Miklowitz, J. 1978. The theory of elastic waves and waveguides. Amsterdam, North-Holland.

Paterson, W.S.B. 1981. The physics of glaciers. Second edition. Oxford, etc., Pergamon Press. (Pergamon International Library.)

Post, A. 1969. Distribution of surging glaciers in western North America. Journal of Glaciology, 8(53), 229-40.

Robin, G. de Q. 1975. Radio-echo sounding: glaciological interpretations and applications. Journal of Glaciology, 15(73), 49-64.

Sheriff, R.E. 1980. Seismic stratigraphy. Boston, International Human Resources Development Corporation.

Tarr, R.S., and Martin, L. 1914. Alaskan glacier studies of the National Geographic Society in the Yakutat Bay, Prince William Sound and lower Copper River regions. Washington, DC, National Geographic Society.

Weertman, J. 1969. Water lubrication mechanism of glacier surges. Canadian Journal of Earth Sciences, 6(4, Pt. 2), 929-42. 